Applied Mathematical Sciences, Vol. 13, 2019, no. 9, 405 - 413

HIKARI Ltd, www.m-hikari.com

https://doi.org/10.12988/ams.2019.9240

\title{
Advertising and Promotion in a Marketing Channel
}

\author{
Alessandra Buratto \\ Dipartimento di Matematica Tullio Levi-Civita \\ Università degli Studi di Padova, Italy \\ Luca Grosset \\ Dipartimento di Matematica Tullio Levi-Civita \\ Università degli Studi di Padova, Italy

\begin{abstract}
In this paper we modify a well-known model about cooperation in a marketing channel in order to obtain a finite horizon linear-state differential game. We obtain that the relevant results about the coordination between the manufacturer and the retailer can be characterised also in our simpler setting. This new approach can be a starting point for further research.
\end{abstract}

This article is distributed under the Creative Commons by-nc-nd Attribution License. Copyright (c) 2019 Hikari Ltd.

Mathematics Subject Classification: 49N90, 91A23, 90B60

Keywords: Differential games, Dynamic advertising, Marketing, Channel coordination.

\section{Introduction}

This work is strongly based on the paper by Jørgensen et al. [5] on the analysis of the coordination between a manufacturer and a retailer in a marketing channel. From a mathematical point of view this model belongs to the class of differential games applied to marketing problems. For a complete introduction about this issue we refer to the book [6]. The differential game we want 
to analyse in the following is based on the Nerlove-Arrow advertising model, which is well-described in the survey [3]. The mathematical tools we want to use in this paper are some standard results about Nash and Stackelberg equilibria in differential games. Our reference for these instruments is the milestone book [1].

We assume, as done in [5], that the marketing channel is composed of a manufacturer (he) and a retailer (she). The manufacturer can drive the evolution of the goodwill of his brand by an advertising campaign (the evolution is based on the Nerlove-Arrow advertising model [3]). The sales function depends on the goodwill and on the promotion which is decided by the retailer. In the first instance of the model advertising costs are sustained by the manufacturer, while promotion costs are sustained by the retailer. In a more interesting instance of the model promotion costs are shared between the manufacturer and the retailer. Under this assumption it is interesting to study when both the players increase their profit; this research question is completely analysed in the paper [5] for a problem with an infinite horizon and with a linear-quadratic structure. Here, we want to simplify the model in two ways: first we assume that the programming interval is finite; second we assume that the structure of the problem is linear-state (see [2]). This simplification is important because it can be a starting point for the analysis of a more complex model which takes into account a marketing segmentation.

In this paper we deal with two main research issues: initially we want to compare the results in a finite horizon with the results in an infinite horizon described in the reference model [5]; then we want to study the impact of the substitution of the linear-quadratic structure with the linear-state one. The results in [5] are the benchmarks for our analysis.

The paper is organised as follows: in Section 2 we describe the model and we compare our instance with the reference instance introduced in [5]. In

Section 3 we assume that there is no coordination between the manufacturer and the retailer i.e. the promotion costs are sustained by the retailer only. In Section 4 we assume that the manufacturer and the retailer sign an agreement: the manufacturer has to share a constant percentage of the retailer's promotion in the whole programming interval. By comparing the payoffs of the players we can identify when this agreement is convenient. Finally, in Section 5, we assume that the sharing percentage of the promotion is a decision variable for the manufacturer. In the Conclusion we illustrate how the results obtained in this paper can be a starting point for further research.

\section{The model}

Let $G$ be the Nerlove-Arrow goodwill, a state function that represents the brand image level of the manufacturer [3]. Goodwill is controlled by the man- 
ufacturer's advertising effort $a \geq 0$, and the following dynamics describe the evolution of the brand image,

$$
\dot{G}=a-\delta G, \quad G(0)=G_{0}>0,
$$

where the parameter $\delta>0$ is the decay rate and $a$ is the control function of the manufacturer. We assume that the sales function is linear with respect to the goodwill:

$$
Q(p, G)=\beta p+G \text {. }
$$

The function $p \geq 0$ represents the promotion effort which is controlled by the retailer ( $\beta>0$ represents the marginal sales with respect to the promotion). We assume both advertising and promotion costs to be quadratic

$$
C_{M}(a)=\kappa_{M} a^{2} / 2, \quad C_{R}(p)=\kappa_{R} p^{2} / 2 .
$$

The advertising costs are sustained by the manufacturer while the promotion costs are sustained by the retailer $\left(\kappa_{M}, \kappa_{R}>0\right.$ are the cost parameters $)$. In order to consider a more general setting we assume that the manufacturer can participate in the promotion expenditure controlled by the retailer. Let us introduce the participation rate $r \in[0,1)$, that represents the percentage of promotion expenditure sustained by the manufacturer; when $r=0$ the manufacturer does not share the retailer's promotion costs. We assume that the programming interval is $[0, T]$, where $T$ is small so that it is not necessary to introduce a discount factor. Under such assumptions the objective function of the manufacturer is

$$
J_{M}(a, r)=\int_{0}^{T} \pi_{M}(\beta p+G)-\kappa_{M} a^{2} / 2-r \kappa_{R} p^{2} / 2 d t,
$$

where the first term under the integral represents the manufacturer's sales profit $\left(\pi_{M}>0\right.$ is the marginal profit), the second term represents the advertising costs, and the third term represents the sharing cost of promotion sustained by the manufacturer. We notice that the participation rate $r$ is considered as a parameter in the manufacturer's objective functional.

The retailer's payoff function is

$$
J_{R}(p, r)=\int_{0}^{T} \pi_{R}(\beta p+G)-(1-r) \kappa_{R} p^{2} / 2 d t,
$$

where the first term under the integral represents the retailer's sales profit $\left(\pi_{R}>0\right.$ is the marginal profit), the second term represents the cost of promotion sustained by the retailer assuming that the manufacturer's participation rate is equal to $r$.

The two objective functionals (9) and (5) together with the motion equation (1) constitute a linear-state differential game. At the end of this section we 
want to focus on the main differences between the reference model proposed in [5] and the simplified version just described. In our model:

- the programming interval is finite $[0, T]$, while in the reference model it is $[0,+\infty)$;

- the discount factor is not taken into account because we assume that $T$ is sufficiently small, while in the reference model both objective functions are described using the discount factor $\exp (\varrho t)$, with $\varrho>0$;

- the sales function is linear in the goodwill, while in the more complete instance of the reference model the sales function is quadratic and concave in the goodwill (this assumption is essential because the Authors want to work with a linear-quadratic differential game in an infinite time horizon);

- the differential game has a linear-state structure, while the reference model has a linear-quadratic structure.

Our model is simpler, but the linear-state structure can be a starting point for an extension that takes market segmentation into account. The same extension is much harder in the reference model. However, before any considerations about this extension, we have to face the solution of the differential game just introduced.

\section{No coordination}

In this Section we assume that the manufacturer does not support the retailer's promotional effort, i.e. the promotion costs are sustained only by the retailer, which means that $r$ is fixed as equal to zero in the formulas of the objective functionals (4) and (5). Without any coordination, it is reasonable to look for a Nash equilibrium. The open-loop Nash equilibrium, which is equivalent to the Markovian Nash equilibrium (see e.g. [2], [4]), is characterised in the following proposition.

Proposition 3.1. (Benchmark) Let us consider the differential game where the manufacturer determines an advertising flow $a \in L^{2}([0, T],[0,+\infty))$ in order to maximise $J_{M}(a, 0)$, while the retailer determines a promotion function $p \in L^{2}([0, T],[0,+\infty))$ in order to maximise $J_{R}(p, 0)$, then the unique openloop Nash equilibrium is

$$
a^{B}(t)=\frac{\pi_{M}}{\kappa_{M}} \frac{\left(1-e^{-\delta(T-t)}\right)}{\delta},
$$




$$
p^{B}(t)=\frac{\pi_{R}}{\kappa_{R}} \beta
$$

Proof. The manufacturer's Hamiltonian function

$$
H_{M}=\pi_{M}(\beta p(t)+G)-\kappa_{M} a^{2} / 2+\lambda_{M}(a-\delta G)
$$

is strictly concave in $a$, hence the optimal advertising strategy is

$$
a^{B}=\lambda_{M}(t) / \kappa_{M},
$$

where the manufacturer's adjoint function $\lambda_{M}(t)$ satisfies the differential equation

$$
\dot{\lambda}_{M}=-\pi_{M}+\delta \lambda_{M}, \quad \lambda_{M}(T)=0 .
$$

Solving (10) and substituting $\lambda_{M}(t)$ in (9) we obtain

$$
a^{B}(t)=\frac{\pi_{M}}{\kappa_{M}} \frac{\left(1-e^{-\delta(T-t)}\right)}{\delta} .
$$

The Hamiltonian (8) is jointly concave in the control and state, hence the necessary conditions are also sufficient.

On the other hand, the retailer's Hamiltonian function

$$
H_{R}=\pi_{R}(\beta p+G)-\kappa_{R} p^{2} / 2+\lambda_{R}(a(t)-\delta G)
$$

is strictly concave in $p$ and its stationary point

$$
p^{B}=\beta \pi_{R} / \kappa_{R}
$$

is optimal because the retailer's Hamiltonian (12) is jointly concave in the control and state, hence the necessary conditions are also sufficient.

Finally, we notice that (11) and (13) are independent, hence they represent the open-loop Nash equilibrium of the differential game.

Without a sharing agreement the differential game degenerates in a couple of unpaired optimal control problems. We notice that the linear-state structure of the model is fundamental to prove that the open-loop Nash equilibrium coincides with the Markovian one.

Comparing our results with the results presented in the reference paper we observe that:

- the optimal promotion strategy of the retailer is exactly the same;

- the optimal advertising strategy of the manufacturer is strictly decreasing in our model (due to the finite programming interval), while it is represented by a linear feedback in the goodwill in the reference model; actually, the instance of the reference model with a linear demand function has a constant optimal advertising strategy which is similar to (6) (i.e. the sensitivity analysis with respect to the parameters are identical in the two models). 


\section{Sharing agreement}

In this section the manufacturer and the retailer can coordinate advertising and promotion in order to improve their profits. At the beginning of the programming interval, the manufacturer and the retailer can sign an agreement which obligates the manufacturer to support the retailer's promotion with a fixed rate $r \in(0,1)$. Moreover, we assume that the agreement is written by a third party and it is mandatory for both players to respect this agreement if it is signed. Hence, both players find their strategies and then compare their optimal profit in the scenario with and without agreement. They sign the agreement if and only if the agreement improves their profits.

Proposition 4.1. (Agreement) Let us consider the differential game where the manufacturer determines an advertising flow $a \in L^{2}([0, T],[0,+\infty))$ in order to maximise $J_{M}(a, r)$, while the retailer determines a promotion function $p \in L^{2}([0, T],[0,+\infty))$ in order to maximise $J_{R}(p, r)$, then the unique openloop Nash equilibrium is

$$
\begin{gathered}
a^{A}(t)=\frac{\pi_{M}}{\kappa_{M}} \frac{\left(1-e^{-(T-t) \delta}\right)}{\delta}, \\
p^{A}=\frac{\pi_{R}}{\kappa_{R}} \frac{\beta}{(1-r)} .
\end{gathered}
$$

The agreement always improves the retailer's profit while it improves the manufacturer's profit if and only if

$$
r<\left(1-\pi_{R} / 2 \pi_{M}\right)
$$

Proof. The manufacturer's Hamiltonian function

$$
H_{M}=\pi_{M}(\beta p(t)+G)-\kappa_{M} a^{2} / 2-r \kappa_{R} p^{2}(t) / 2
$$

depends additively on the promotion (retailer's control), hence the optimal advertising strategy remains (9). The retailer's Hamiltonian function is

$$
H_{R}=\pi_{R}(\beta p+G)-(1-r) \kappa_{R} p^{2} / 2+\lambda_{R}(a(t)-\delta G),
$$

hence, as done in the proof of Proposition 3.1, we obtain

$$
p^{A}=\frac{\pi_{R} \beta}{\kappa_{R}(1-r)} .
$$

After substituting the optimal advertising and promotion in the objective functionals of the manufacturer and of the retailer we obtain 


$$
J_{R}\left(p^{A}, r\right)-J_{R}\left(p^{B}, r\right)=\frac{\pi_{R}^{2} T \beta^{2} r}{2 \kappa_{R}(1-r)}
$$

and

$$
J_{M}\left(a^{A}, r\right)-J_{M}\left(a^{B}, r\right)=\left(2 \pi_{M}(1-r)-\pi_{R}\right) \frac{\pi_{M} T \beta^{2} r}{2 \kappa_{M}(1-r)^{2}} .
$$

The retailer's profit difference is always positive while the manufacturer's one is positive if and only if (16) holds.

\section{$5 \quad$ Stackelberg game}

The main result of the previous section is that the retailer's profit always improves when the agreement is signed. Therefore the manufacturer can propose an agreement to the retailer acting as a leader in a Stackelberg differential game where the retailer is the follower. Under this hypothesis the manufacturer can not only control the advertising, but can also decide the participation rate in the retailer's promotion. This means assuming that the participation rate is a manufacturer's control variable.

Proposition 5.1. (Stackelberg)Let us consider the Stackelberg differential game where the manufacturer (leader) determines an advertising flow a $\in$ $L^{2}([0, T],[0,+\infty))$ and the participation rate $r \in L^{2}([0, T],[0,1])$ in order to maximise $J_{M}(a, r)$ while the retailer (follower) determines a promotion function $p \in L^{2}([0, T],[0,+\infty))$ in order to maximise $J_{R}(p, r)$. Then the unique time-consistent Stackelberg equilibrium is

$$
\begin{gathered}
p^{S}=\frac{\pi_{R} \beta}{\kappa_{R}\left(1-r^{S}\right)}, \\
a^{S}(t)=\frac{\pi_{M}}{\kappa_{M}} \frac{\left(1-e^{-(T-t) \delta}\right)}{\delta}, \\
r^{S}=\max \left\{\frac{2 \pi_{M}-\pi_{R}}{2 \pi_{M}+\pi_{R}}, 0\right\} .
\end{gathered}
$$

Proof. To compute a Stackelberg equilibrium, we first determine the reaction function of the retailer to the manufacturer's strategies and then we solve the manufacturer's optimal control problem. The retailer's Hamiltonian function

$$
H_{R}=\pi_{R}(\beta p+G)-(1-r(t)) \kappa_{R} p^{2} / 2+\lambda_{R}(a(t)-\delta G)
$$

is strictly concave in $p$, hence the promotion strategy

$$
p^{S}=\frac{\pi_{R} \beta}{\kappa_{R}(1-r(t))}
$$


is optimal because the retailer's Hamiltonian (23) is jointly concave in the control and state, hence the Mangasarian sufficiency conditions hold.

If we substitute the retailer's best response strategy into the manufacturer's Hamiltonian function, we obtain

$$
H_{M}=\pi_{M}\left(\frac{\pi_{R} \beta^{2}}{\kappa_{R}(1-r)}+G\right)-\kappa_{M} a^{2} / 2-r \frac{\pi_{R}^{2} \beta^{2}}{2 \kappa_{R}(1-r)}+\lambda_{M}(a-\delta G) .
$$

Deriving with respect to the controls $a$ and $r$ we have

$$
\left(\frac{\partial H_{M}}{\partial a}, \frac{\partial H_{M}}{\partial r}\right)=\left(-a \kappa_{M}+\lambda_{M}, \frac{\pi_{R}\left(2 \pi_{M}(1-r)-\pi_{R}(1+r)\right) \beta^{2}}{2 \kappa_{R}(1-r)^{3}}\right) .
$$

Recalling that $r \in(0,1)$ we obtain the promotion strategy (22)

$$
r^{S}=\max \left\{\frac{2 \pi_{M}-\pi_{R}}{2 \pi_{M}+\pi_{R}}, 0\right\}
$$

and the advertising strategy

$$
a^{S}(t)=\frac{\lambda_{M}(t)}{\kappa_{M}}
$$

In order to obtain the advertising strategy we solve the adjoint equation, which coincides with (10), so that the advertising Stackelberg equilibrium strategy is (21). Once placed the optimal controls (22) and (21) in the Hamiltonian function we can verify that Arrow's sufficiency theorem holds, so that the obtained strategies constitute the optimal Stackelberg equilibrium.

We notice that the analytical form of the Stackelberg equilibrium is equivalent to the analytical form we have already discussed at the end of Section 4. Hence, the retailer always gains a higher profit when the manufacturer participates in the promotion expenditure, and, under the hypotheses of this section, it happens if and only if $\left(2 \pi_{M}-\pi_{R}\right)>0$. As far as the manufacturer is concerned, his profit increases if and only if $r^{S}$ satisfies (16) and this trivially holds true when $\left(2 \pi_{M}-\pi_{R}\right)>0$.

It is interesting to notice that, even if our model is simpler, our solutions are the same as the ones presented in the reference paper [5] (the unique difference is in the optimal advertising flow, and it is connected to the different horizon of the two models). 


\section{Conclusion}

In this paper we modify a well-known model about cooperation in a marketing channel [5] and we study how our different assumptions change the reference results. From a mathematical point of view, the reference model is an infinite horizon linear-quadratic differential game, while our model is a linear-state differential game. We obtain that the results of the two models are comparable and, for many aspects, they are the same. This is important because further research could consider market segmentation and facing this issue with a simpler model is fundamental in order to obtain explicit solutions.

\section{References}

[1] E. J. Dockner, S. Jørgensen, N. Van Long and G. Sorger, Differential Games in Economics and Management Science, Cambridge University Press, Cambridge, 2000. https://doi.org/10.1017/cbo9780511805127

[2] L. Grosset, A Note on Open Loop Nash Equilibrium in Linear-State differential games, Applied Mathematical Sciences, 8(145) (2014), 7239 - 7248. https://doi.org/10.12988/ams.2014.49746

[3] J. Huang, M. Leng, L. Liang, Recent developments in dynamic advertising research, European Journal of Operational Research, 220(3) (2012), 591609. https://doi.org/10.1016/j.ejor.2012.02.031

[4] S. Jørgensen, G. Martın-Herran, and G. Zaccour, The LeitmannSchmitendorf advertising differential game, Applied Mathematics and Computation, 217(3) (2010), 1110-1116.

https://doi.org/10.1016/j.amc.2010.01.047

[5] Jørgensen, S. Taboubi and G. Zaccour, Cooperative Advertising in a Marketing Channel, Journal of Optimization Theory and Applications, 110(1) (2001), 145-158. https://doi.org/10.1023/A:1017547630113

[6] S. Jørgensen and G. Zaccour, Differential Games in Marketing, Kluwer Academic, Boston, 2004. https://doi.org/10.1007/978-1-4419-8929-1

Received: March 11, 2019; Published: April 11, 2019 